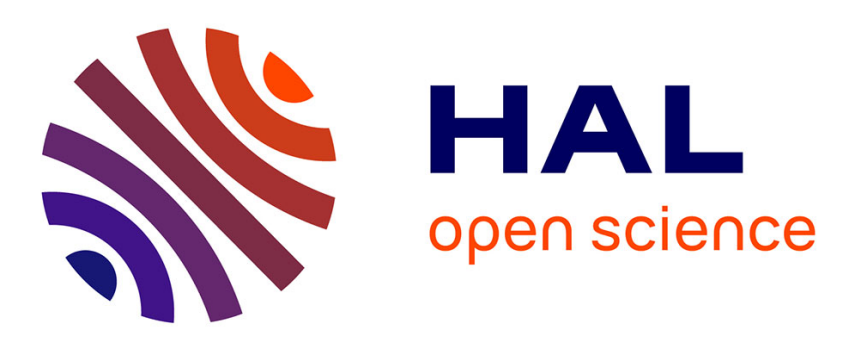

\title{
Mixed Reality Navigation on a Tablet Computer for Supporting Machine Maintenance in Wide-area Indoor Environment
}

Koji Makita, Thomas Vincent, Soichi Ebisuno, Masakatsu Kourogi, Tomoya

Ishikawa, Takashi Okuma, Minoru Yoshida, Laurence Nigay, Takeshi Kurata

\section{To cite this version:}

Koji Makita, Thomas Vincent, Soichi Ebisuno, Masakatsu Kourogi, Tomoya Ishikawa, et al.. Mixed Reality Navigation on a Tablet Computer for Supporting Machine Maintenance in Wide-area Indoor Environment. 2nd International Conference on Serviceology, Sep 2014, Yokohama, Japan. pp.109-124, 10.1007/978-4-431-55861-3_8. hal-01492559

\section{HAL Id: hal-01492559 \\ https://hal.science/hal-01492559}

Submitted on 20 Mar 2017

HAL is a multi-disciplinary open access archive for the deposit and dissemination of scientific research documents, whether they are published or not. The documents may come from teaching and research institutions in France or abroad, or from public or private research centers.
L'archive ouverte pluridisciplinaire HAL, est destinée au dépôt et à la diffusion de documents scientifiques de niveau recherche, publiés ou non, émanant des établissements d'enseignement et de recherche français ou étrangers, des laboratoires publics ou privés. 


\title{
Mixed Reality Navigation on a Tablet Computer for Supporting Machine Maintenance in Wide-area Indoor Environment
}

\author{
Koji Makita1, Thomas Vincent², Soichi Ebisuno ${ }^{3}$, Masakatsu Kourogi11,4, Tomoya Ishikawa4, \\ Takashi Okuma1, Minoru Yoshida ${ }^{3}$, Laurence Nigay², and Takeshi Kurata1 \\ ${ }^{1}$ Center for Service Research, National Institute of Advanced Industrial Science and Technology, \\ AIST Tsukuba Central 2, 1-1-1, Umezono, Tsukuba, Ibaraki 305-8568, Japan \\ Tel: +81 29862 6734, Fax: +81 29862 6548, E-mail: k.makita@aist.go.jp \\ 2 Université Joseph Fourier, France \\ ${ }^{3}$ Digital Electronics Corporation $\quad{ }^{4}$ KODO Lab Inc.
}

\begin{abstract}
This paper describes a maintenance service support system for wide-area indoor environment, such as a factory and a hospital. In maintenance services, operators often have to check a map to find out a way to a target machine, and also have to refer documents to get information about check-up and repair of the machine. In order to reduce working load of operators, information technology can help operators carry out additional but important operations during maintenance, such as referring documents and maps, recording maintenance logs and so on. In this paper, we propose mixed reality navigation on a tablet computer composed of augmented virtuality mode and augmented reality mode. Augmented virtuality mode performs map-based navigation shows positions of the user and the target machine. Augmented reality mode performs intuitive visualization of information about the machine by overlaying annotations on camera images. The proposed system is based on a hybrid localization technique realized with pedestrian dead reckoning (PDR) and 3D model-based image processing for the purpose of covering wide-area indoor environment. Experimental results using our prototype with a mock-up model of a machine are also described for showing feasibility of our concept in the paper.
\end{abstract}

\section{Keywords:}

Maintenance support, Human navigation, Mixed reality, Mobile computing

\section{INTRODUCTION}

Machine maintenance services are essential for workers to safely and efficiently use machines. For maintenance operators, main activities of maintenance services are to go in front of the target machine, and check-up of the machine. Moreover, in case failure parts are detected, additional activities such as doing repairs, component replacement and orders for components are occurred.

Because maintenance services are not routine, the activities can be usually inexperienced works. Therefore, maintenance services can be difficult and time-consuming because they require lots of information. Operators often have to check a map to find out a way to a target machine, and also have to refer documents to get information about check-up and repair of the machine. Therefore, especially in wide-area indoor environment, such as a factory and a hospital, working load of operators should be reduced.

In this paper, we propose a maintenance service support system for wide-area indoor environment to reduce working load of operators. We focus on working load of obtaining information. In order to realize the system to reduce working load of obtaining information, we introduce mixed reality (MR) concept. MR is an inclusive term of techniques to merge virtual and real world. Since MR has a possibility to realize intuitive information presentation, we propose mixed reality navigation on a tablet computer composed of augmented virtuality mode and augmented reality mode. Augmented virtuality (AV) and Augmented Reality (AR) are components of MR. Augmented virtuality mode performs map-based navigation shows positions of the user and the target machine. Augmented reality mode performs intuitive visualization of information about the machine by overlaying annotations on camera images.

\section{RELATED WORKS}

How MR can assist in reducing the time and effort in maintenance is evaluated in previous works [1] [2]. The majority of related work focuses on MR applications work in front of the target object. On the contrary, we focus on how to realize localization in wide-area indoor environment for MR.

To achieve robust and global localization in wide areas for mobile MR, one solution is to combine a method works constantly and the visual tracking method, because visual tracking can be used for precisely estimating position and posture [3] only in case a camera of mobile device is active.

GPS is often used for initialization [4] [5]. While GPS can provide global position and posture without any previous knowledge, it works only in outdoor environments. On the other hand, for indoor MR, several approaches requiring previous knowledge have been proposed, one of which is the marker based localization method. This method can be used in environments with marker infrastructure already in place [6]. Therefore, these methods are appropriate for constructing a special environment or desktop application that works in small spaces.

Marker based methods can be used for initialization only when markers are captured by a camera. For more efficient construction of a wide MR space, there exist devices that can be applied to construct positioning infrastructure. For example, the radio frequency identification RFID and infrared data association IrDA based methods [7] have been proposed. On the other hand, a positioning system using wireless LAN has also been proposed [8]. These methods are effective for acquiring an approximate global position in large indoor environments. However, the accuracy of these methods is not normally adequate for the initialization of visual tracking methods. Image based matching methods [9] [10] 
[11] can also be applied for initialization. However, because these methods use 2D image based matching, many reference images are needed to cover large environments and for accurate initialization. On the other hand, in existing tracking methods using 3D models [12] [13], a matching between a captured image and an image generated from models is used to estimate relative position and posture.

Recently, 3D reconstruction methods have been proposed. For large scale accurate reconstruction, modeling methods using robots with range sensors are effective [14] [15]. Moreover, interactive image-based modeling methods have been proposed [16] [17] [18] [19].

These methods encourage more convenient 3D reconstruction, and can be applied to various environments. For example, Figure 1 shows a sample generated image from 3D models generated using an interactive 3D modeler [18]. Once models are generated, images from any views can be obtained. Therefore, models can be applied various applications. Figure 2 shows a sample image of augmented virtuality (AV) application. In a scene 3D dataset (point features, edgelets, etc.) can be obtained, global position and posture of a device are estimated with the dataset [20] [21] [22]. But normally generation cost of the $3 D$ dataset is higher than the one of 3D models in terms of a number of images needed to be generated. Therefore, the estimation using 3D models has possibility to efficiently extend areas for mobile MR.

This study focuses on the ability to merge pedestrian dead reckoning (PDR) and 3D model-based image processing for the purpose of covering wide-area indoor environment. PDR is a technique for the measurement of position and orientation based on dead reckoning that focuses on human walking motion detection. Because the PDR is normally realized with self-contained wearable or mobile sensors, localization can always work. The PDR works well for application systems such as humannavigation systems which user is mainly walking during using the system. However, the positioning accuracy of the PDR is often getting down in working situation, and includes accumulative errors. On the contrary, image processing can work only in case a camera of mobile device is active and quality of camera images are well. For instance, blur of the images and less image features affect accuracy of the localization. However, normally the accuracy of the localization is high, and accumulative errors can be reduced with key-frame based initialization.

\section{PROTOTYPE}

Figure 3 shows an overview of the proposed localization method. Position and orientation of the user of a tablet computer can be always estimated with PDR. However, the positioning accuracy is getting down with the accumulative errors because of various kinds of actions except for walking. For creating a prototype system in this paper, we implemented a PDR application based on a method shown in [23] on a smart phone (Samsung RZ Galaxy). Acceleration sensors, angular velocity sensors, and magnetic sensors in the smart phone are used for the estimation procedure of PDR. The smart phone is attached to the waist of the user, and position and orientation data estimated by the PDR application is always sent to the tablet computer via wireless network. Only with PDR, the accumulative errors are not able to be reduced.

On the other hand, when the camera of the tablet computer is active, comparisons of camera image and images used for creating 3D model are conducted. Figure 4 shows an overview of the comparisons. Afterward, we call this comparison "key frame matching". Images used for creating 3D model are photos with known photo-shoot
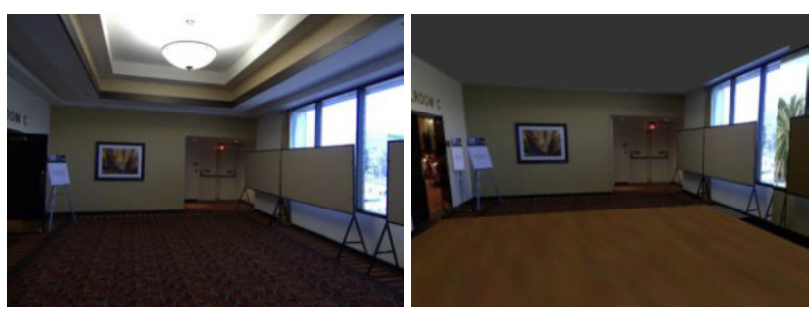

Fig. 1: An example of an input image and a corresponding image from models. (Left: Input image taken in indoor environment. Right: Corresponding image from models that are generated using an interactive $3 D$ modeler [18].)
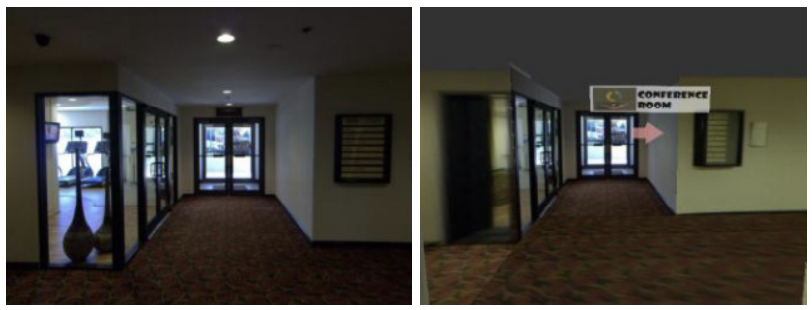

Fig. 2: Left: Input image taken in indoor environment. Right: Screen shot of augmented virtuality (AV) application. This generated image is composed of an image from models and navigation information.

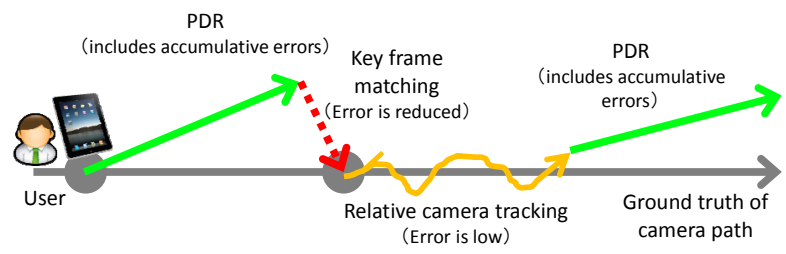

Fig. 3 : Overview of the localization method.

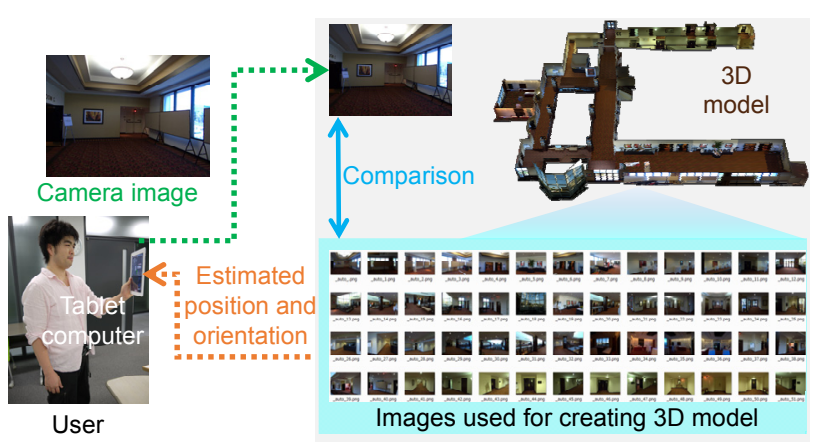

Fig. 4 : Overview of the key frame matching.

positions and orientations, and depth data. For creating a prototype system in this paper, we implemented an application for (re-)initialization based on a method shown in [24] on a tablet computer (Apple iPad2). Because of applying secondary product of modeling process, additional works are not necessary for set up. In the method, an image of real environment taken by a mobile camera of the tablet computer is compared to images used for creating 3D model. Because images used for creating 3D model are linked with photo-shoot position, orientation, and depth data, 3D coordinates of each pixel on the images is available in the model coordinate system. Therefore, various types of camera tracking method using image features, such as feature points and edges, are able to be applied to the localization. For the 
prototype, we applied an estimation technique using feature point-matching method, and selected Speeded Up Robust Features (SURF) [25] for the detection of feature points. Normally, the calculation time of the matching procedure is too long to be applied to MR application. Therefore, after the matching is succeeded, image processing procedure is changed to a relative camera tracking. Since normally the relative camera tracking can also be realized with image features, in this paper, we applied Lucas-Kanade-Tomasi (LKT) trackers shown in [26].

When the relative camera tracking fails, the PDR application is used again. Currently, for re-initializing the PDR, the last result of the relative camera tracking is simply applied, because the accuracy of relative camera tracking is supposed to be higher than that of the PDR in most cases. However, since there is a case to get errors of the key frame matching and relative camera tracking, we have the possibility of improvement in re-initialization of the PDR. After that, the key frame matching also work again.

\section{EXPERIMENTS WITH THE PROTOTYPE}

We conducted an experiment in our office building in order to check the behavior of the prototype system. In the experiment, we evaluated a moving distance when the key frame matching is succeeded. Motivation of the evaluation is below.

Figure 5 shows samples of moving distances when the key frame matching is succeeded. As shown in Figure 4, when the key frame matching is conducted, a camera image captured by the tablet computer is compared to images used for creating 3D model. Generally, we have lots of images used for creating 3D model, and retrieve the images in distance order from the position estimated by PDR. Therefore, the computational time of the key frame matching is proportional to the moving distance. Fundamentally, lots of images should be used, and the computational time should be measured. However, since currently we do not have any speeding up method in the retrieval, there is a high possibility of exceedingly-long computational time. Therefore, in this experiment, we preliminarily set only one image as a target of the key frame matching, and measured the moving distance.

Experimental set up is below. Figure 6 shows appearances of the user and the AVIAR application. First, we created a virtualized reality model of our office building with the method shown in [18], and prepare one image for the key frame matching. Next, we set a round course in the building whose distance is about 60 meters, and set one user as a subject. In the experiment, at first, the user stands at the start position of the course, and hold the tablet computer to conduct the key frame matching with AR mode. After key frame matching is succeeded, the user walks along the course with AV mode. Finally, the user stands at the start position and conduct the key frame matching with $A R$ mode again, and the moving distance of latter matching is measured. In order to check difference between walking distance and the moving distance of latter matching, we conducted experiments five times with one cycle (about 60 meters) and two cycles (about 120 meters).

Figure 7 shows examples of localization results. In these figures, blue lines indicate the moving distance of former matching occurred because the difference of manual initialization and the start point. Red lines indicate the pass when the method is changed from the relative camera tracking to PDR. Sky blue lines indicate the pass estimated with PDR. Finally, green lines indicate the moving distance of latter matching we focus on in the experiments.

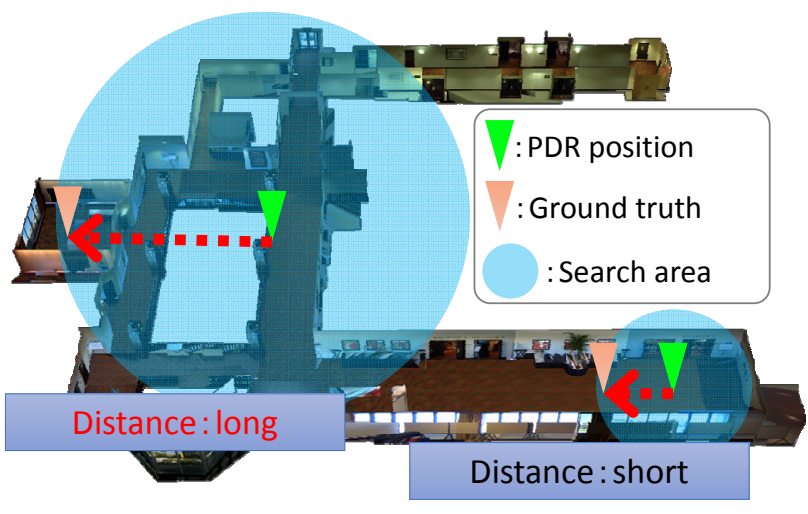

Fig. 5 : Moving distances when the key frame matching is succeeded.
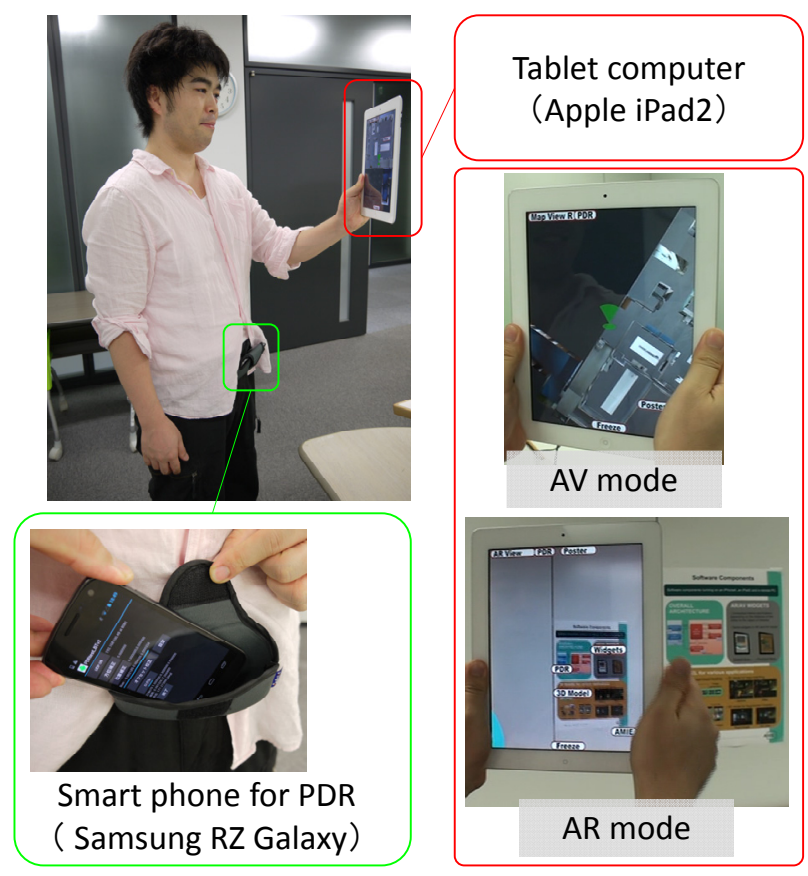

Fig. 6: Appearances of the user and the AV/AR application.

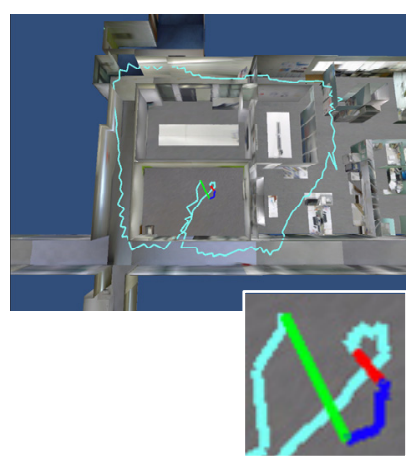

(a)

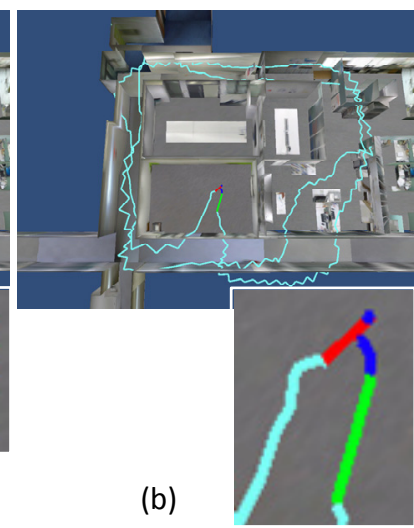

(b)
Fig. 7 : Examples of localization results. (a) one cycle, (b) two cycles. Each green line indicates the moving distance of latter matching.

Figure 8 shows all results of the experiments. In the results, average moving distance of latter matching was about 1.44 meter with one cycle, and about 2.03 meter with two cycles. 


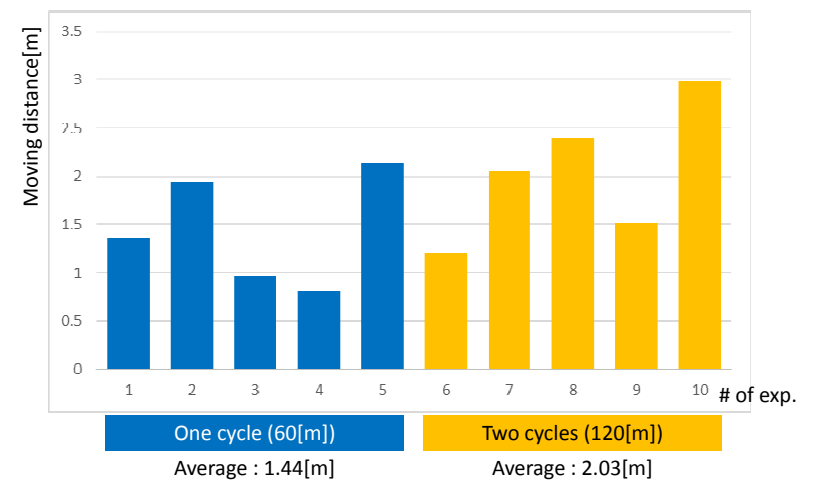

Fig. 8 : Moving distances of latter matching.

As a result, we have successfully checked the behaviour of the prototype system. Because we have the variability of walking motion, the moving distances of latter matching also have the variability. In terms of the average, however, the results shown in Figure 8 are reasonable because basically the error of PDR is proportional to the walking distance. In future, in case we use lots of images for the key frame matching, we have to consider about the trade-off between the number of chances of the matching and the computational time. Specifically, spatial configuration and resolution of the images for the matching should be optimized. In the experiment, we set the resolution at $240 * 180$, and the computational time was about $1-2$ seconds.

Moreover in the future, we plan to conduct subjective experiments with a mock-up model of a machine. Figure 9 shows appearances of the tablet computer (Toshiba dynabook Tab for weight saving) and the mock-up. In order to study the effectiveness, we have been implementing both AV and AR applications to effectively create and show annotations with precise pointing techniques shown in [27]. In the experiments of this section, AV mode was only used for the map based navigation. However, we already have implemented both $A R$ and $A V$ mode to showing annotations. In experiments described in Section 5, we apply AV mode to show annotations, and conduct subjective experiments.

\section{IN-FIELD EVALUATION}

We conducted customer interest check $(\mathrm{CIC})$ as in-field evaluations. The goal of the evaluations is to survey the acceptability, usefulness and usability of the prototype system for engineers and operators working in a factory. The evaluations were conducted with six maintenance engineers and operators working in France and six maintenance engineers and operators working in Japan. Procedures of evaluations are below.

\section{Presentation of the concept and the movie}

Each interviewee watches a movie for showing the concept of the proposed system. Interviewers supplement the explanation of the movie with their talking when the interviewee has questions about the proposed system.

\section{Evaluation of the acceptability}

Each interviewee is asked 20 questions of CAUTIC [28] about the proposed system, and choose the one answer from "Acceptable", "Acceptable under conditions" and "Not acceptable". CAUTIC is an interview method for evaluating an acceptability of a product. CAUTIC has scientifically identified 20 criteria at 4 levels of analysis (technical, practical, user identity and user environment)
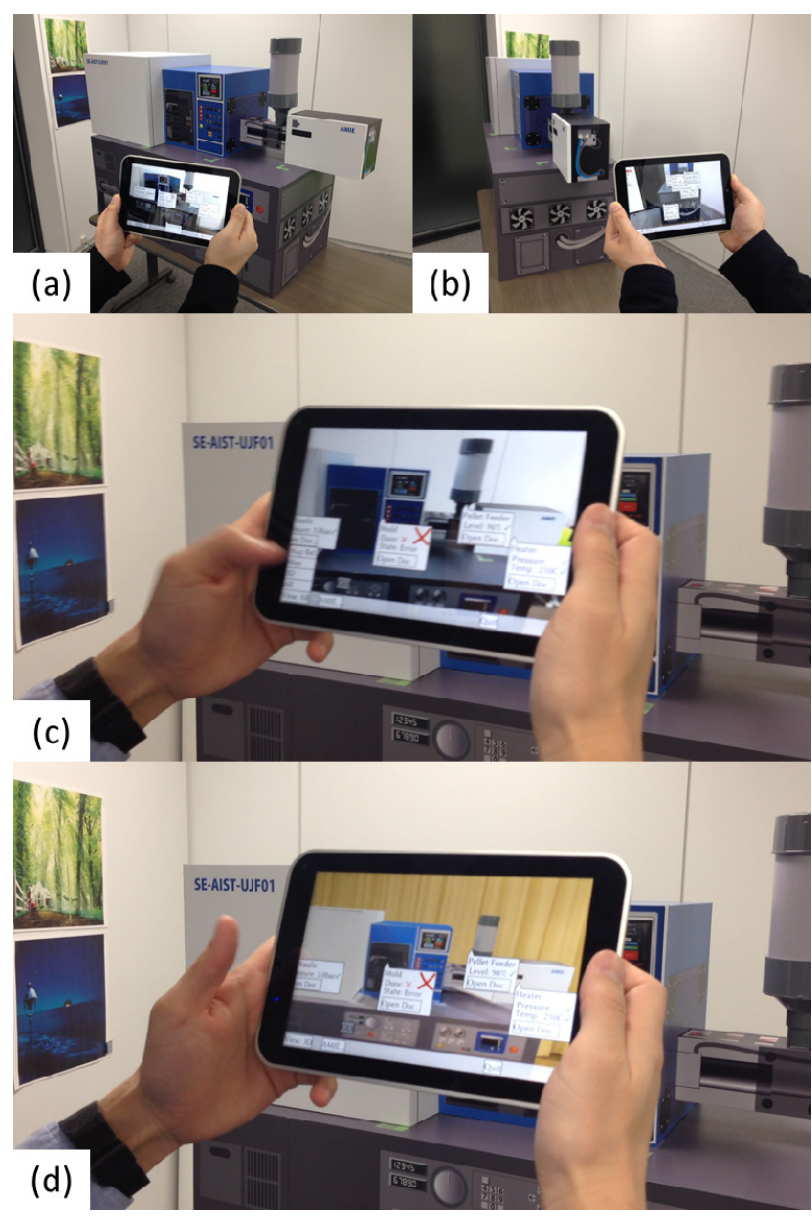

Fig. 9 : Appearances of the tablet computer and the mock-up. (a) and (b): Overviews of the mock-up. (c): Annotation overlay with AR mode. (d): Annotation overlay with AV mode.

that must be validated in order to permit to establish, beyond doubt, whether or not the compelling reason to buy exists. If the 20 criteria are not or only partially validated, the method identifies the problem areas from each of the 4 levels that obstruct acceptance of the innovation in question. These areas can be subsequently given remedial treatment, which in turn can be verified by the CAUTIC method if seemed necessary.

\section{Evaluation of usefulness}

Each interviewee is asked a couple of questions about each function of the proposed system from KANO method [29]. In KANO method, each question is composed of a functional and dysfunctional questions, and the interviewee choose the one answer from "Like", "Must be", "Neutral", "Live with" and "Dislike". By combining the two answers, quality attributes are grouped into six categories (Attractive, One-dimensional, Must be, Questionable, Reverse, Indifferent) with a different impact on customer satisfaction [30].

\section{Results of the evaluations are shown below.}

\section{Acceptability}

Table 1 indicates CAUTIC's questions and of results of six respondents in France and Japan. The number of stars $\left({ }^{*}\right.$ in the right side of Table 1$)$ is decided with an average score $M$ of the answers. We set scores $(2,1,0)$ for "Acceptable", "Acceptable under conditions" and "Not acceptable" respectively to calculate $M\left(M>=1.33:{ }^{* * *}\right.$, $\left.0.67<=M<1.33:{ }^{* *}, M<0.67:{ }^{*}\right)$. Totally, the evaluation 
of the acceptability by CAUTIC reveals that the proposed system is well received by maintenance technicians and managers. In the results of France, average score $M$ of 1.2 and 2.5 are comparatively low. In 1.2 , there were several questions about how to connecting the machine to get information of the machine, and about scalability of the system. In 2.5, there were several questions about capability of false recognition of the machine, and about capacity to update technical documentations. In the results of Japan, average score $M$ of 3.2 and 4.3 are comparatively low. The main reason of these results were interviewees' impression of the proposed system as their private role. Two interviewees answered "Not acceptable" for 3.2 and 4.3 because they do not have any ideas of using the proposed system privately.

As was pointed out in the results of France, scalability and the capability of false recognition are very important in terms of the comfort of the system. Experiments in wide area with multiple machines are next steps of this study. Required time and false recognition rate of the key frame matching are supposed to become progressively higher with an increase in the number of key frames. In future, we plan to study fast search of the key frames. Humancomputer interaction technique is one way to realize the fast search. For example, showing candidates of the key frames is supposed to be worth to shorten the required time of the image matching.

\section{Usefulness :}

Table 2 indicates functions of the proposed system, and KANO's evaluation table of six respondents in France and Japan. First, in France, we applied eight questions for the KANO method. Subsequently, in Japan, we added two functions: "Freeze function" and "Visualization of the machine in Augmented Virtuality" to the system, and applied ten questions for the KANO method. "Freeze functions" is a function to temporarily stop updating the displayed image of AR/AV mode. We assume "Freeze functions" is helpful as hands-free capability. "Visualization of the machine in Augmented Virtuality " is a function to overlay annotations with AV mode as shown in Figure 9(d).

Dim1 and Dim2 indicate first and second components of the category of the answers (A: Attractive, M: Must-be, R: Reverse, O: One-dimensional, Q: Questionable, I: Indifferent, $\mathrm{N}$ : None) with the number of the answers. For example, "Q(5)" indicates that there are five answers categorised in "Questionable". In case the number of the category of multiple answers is one, we applied "None" as the component of the category. In the results of France, functions 4(Access to the diagnosis), 5(Technical documentation), 7 (Contents of the electric cabinet without having to open the machine) and 8(Creation of maintenance report) were categorised in " Attractive" as first component. In the results of Japan, 2(Guidance toward the machine), 4(Access to the diagnosis) and 10 (Visualization of the machine in Augmented Virtuality) were categorised in " Attractive" as first component.

Throughout the whole experiments, interviewees in Japan had more previous knowledge of AR than interviewees in France, and there were several answers categorised in "Questionable" only in France. 1(Visualization of the machine in Augmented Reality) was categorised in "Questionable" as first component in France. This is due to the difficulty of the users to imagine AR function before using it. Five interviewees could not understand the difference of the way of provision of information. Therefore, they selected the same answer for the both questions. Moreover, in the results in France, 4(Access to the diagnosis) and 5(Technical documentation) were also categorised in "Questionable" as second component. These are also probably due to the difficulty to imagine AR function. On the contrary, in Japan, there were not any answers categorised in "Questionable".

\section{CONCLUSION}

This paper has proposed a maintenance service support system for wide-area indoor environment. In order to realize MR maintenance service support system, we proposed a hybrid localization technique realized with pedestrian dead reckoning (PDR) and 3D model-based image processing for the purpose of covering wide-area indoor environment. As a first step of the realization, this paper presents experimental results that show the relation between the walking distance and the moving distance of the key frame matching that is strongly related to the computational time of the key frame matching. As regards future works, we plan to study the optimization of the key frame matching to realize appropriate accuracy and computational time of the localization, and subjective experiments with both mock-up and real machine in wide indoor environments.

\section{ACKNOWLEDGEMENTS}

This work was supported by Strategic Japanese-French Cooperative Program on Information and Communication Technology Including Computer Science (ANR in France and JST in Japan).

Table 1. Results of CAUTIC

\begin{tabular}{|c|c|c|c|}
\hline & & France & Japan \\
\hline \multirow{5}{*}{ 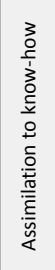 } & 1.1. Does the concept interest the user in principle? & $* * *$ & $* * *$ \\
\hline & $\begin{array}{l}\text { 1.2. Is the concept's technical principle understandable } \\
\text { enough for the user? }\end{array}$ & $* *$ & $* * *$ \\
\hline & $\begin{array}{l}\text { 1.3. Can the user connect / compare the concept } \\
\text { techniques to existing techniques? }\end{array}$ & $* * *$ & $* * *$ \\
\hline & $\begin{array}{l}\text { 1.4. Can the user easily identify and use the function(s) } \\
\text { which interest him in the concept? }\end{array}$ & $* * *$ & $* * *$ \\
\hline & $\begin{array}{l}\text { 1.5. Is the technical principle of the concept simple } \\
\text { enough to be used at anytime? }\end{array}$ & $* * *$ & $* * *$ \\
\hline \multirow{5}{*}{ 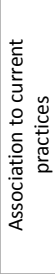 } & 2.1. Does the concept seem simple to use for the user? & $* * *$ & $* * *$ \\
\hline & $\begin{array}{l}\text { 2.2. Does the concept allow to complete current practices, } \\
\text { without interfering with them? }\end{array}$ & $* * *$ & $* * *$ \\
\hline & $\begin{array}{l}\text { 2.3. Does the comparison with the current practices } \\
\text { validate and make credible the new suggested practices? }\end{array}$ & $* * *$ & $* * *$ \\
\hline & $\begin{array}{l}\text { 2.4. Does the concept solve at least one practical problem } \\
\text { that the user is facing? }\end{array}$ & $* * *$ & $* * *$ \\
\hline & $\begin{array}{l}\text { 2.5. Can the user gradually foresee a new personal } \\
\text { organization of his practices by using the concept? }\end{array}$ & $* *$ & $* * *$ \\
\hline \multirow{5}{*}{ 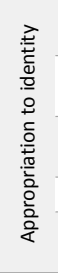 } & $\begin{array}{l}\text { 3.1. Does the user recognize himself as the targeted user } \\
\text { and know who else the concept concerns? }\end{array}$ & $* * *$ & $* * *$ \\
\hline & $\begin{array}{l}\text { 3.2. Can the user use the concept to act on his private / } \\
\text { professional role? }\end{array}$ & $* * *$ & $* *$ \\
\hline & $\begin{array}{l}\text { 3.3. Is the concept compatible with the user's personal / } \\
\text { professional values? }\end{array}$ & $* * *$ & $* * *$ \\
\hline & 3.4. Does the user appropriate the concept? & $* * *$ & $* * *$ \\
\hline & $\begin{array}{l}\text { 3.5. Does the user imagine alternative or extended uses } \\
\text { to the concept? }\end{array}$ & $* * *$ & $* * *$ \\
\hline \multirow{5}{*}{ 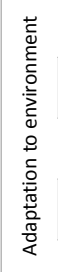 } & $\begin{array}{l}\text { 4.1. Is the concept arriving on the market at the right } \\
\text { time? }\end{array}$ & $* * *$ & $* * *$ \\
\hline & $\begin{array}{l}\text { 4.2. Is the concept adapted to the user's client-supplier / } \\
\text { family relations evolution? }\end{array}$ & $* * *$ & $* * *$ \\
\hline & $\begin{array}{l}\text { 4.3. Is the concept adapted to the user's position in } \\
\text { his/her professional / private circle? }\end{array}$ & $* * *$ & $* *$ \\
\hline & $\begin{array}{l}\text { 4.4. Is the concept adapted to the user's working } \\
\text { organization/ way of living and its evolution? }\end{array}$ & $* * *$ & $* * *$ \\
\hline & 4.5. Does the user agree to pay for the concept? & $* * *$ & $* * *$ \\
\hline
\end{tabular}


Table 2. Results of KANO

\begin{tabular}{|c|c|c|c|c|}
\hline \multirow{2}{*}{ Functions of the proposed system } & \multicolumn{2}{|c|}{ France } & \multicolumn{2}{|c|}{ Japan } \\
\hline & $\operatorname{Dim} 1$ & $\operatorname{Dim} 2$ & Dim1 & Dim2 \\
\hline $\begin{array}{l}1 \text { : Visualization of the machine in } \\
\text { Augmented Reality }\end{array}$ & $Q(5)$ & $R(1)$ & $\mathrm{O}(3)$ & $A(2)$ \\
\hline $2:$ Guidance toward the machine & I(3) & $A(2)$ & $A(4)$ & $\mathrm{O}(2)$ \\
\hline 3 : Maintenance history & $\mathrm{I}(2)$ & $A(2)$ & $\mathrm{O}(3)$ & $A(2)$ \\
\hline $4:$ Access to the diagnosis & $A(4)$ & $Q(2)$ & $A(3)$ & $\mathrm{O}(2)$ \\
\hline 5 : Technical documentation & $A(3)$ & $Q(2)$ & $\mathrm{O}(2)$ & $A(2)$ \\
\hline $\begin{array}{l}6: \text { Access to parts inventory } \\
\text { replacement }\end{array}$ & $\mathrm{O}(2)$ & $\mathrm{N}$ & $\mathrm{O}(4)$ & $\mathrm{N}$ \\
\hline $\begin{array}{l}7: \text { Contents of the electric cabinet } \\
\text { without having to open the machine }\end{array}$ & $A(3)$ & I(3) & $\mathrm{O}(3)$ & $\mathrm{N}$ \\
\hline $8:$ Creation of maintenance report & $A(4)$ & $\mathrm{N}$ & $\mathrm{O}(4)$ & $\mathrm{N}$ \\
\hline 9 : Freeze function & --- & --- & $\mathrm{O}(4)$ & $\mathrm{N}$ \\
\hline $\begin{array}{l}10: \text { Visualization of the machine in } \\
\text { Augmented Virtuality }\end{array}$ & --- & --- & $A(3)$ & $\mathrm{I}(2)$ \\
\hline
\end{tabular}

\section{REFERENCE}

[1] Henderson S. J and Feiner S, 2009, Evaluating the Benefits of Augmented Reality for Task Localization in Maintenance of an Armored Personnel Carrier Turret, IEEE International Symposium on Mixed and Augmented Reality 2009, Science and Technology Proceedings: 135-144.

[2] Platonov J, Heibe $\mathrm{H}$, Meier $\mathrm{P}$ and Grollmann B, 2006, A mobile markerless AR system for maintenance and repair, Proc. of the 5th IEEE and ACM International Symposium on Mixed and Augmented Reality: 105-108.

[3] Klein G and Murray D, 2009, Parallel tracking and mapping on a camera phone, Proc. Int. Symp. on Mixed and Augmented Reality: 83-86.

[4] Reitmayr G and Drummond T, 2007, Initialisation for Visual Tracking in Urban Environments, Proc. Int. Symp. on Mixed and Augmented Reality: 161-172.

[5] Höllerer T, Feiner S, Terauchi T, Rashid G and Hallaway D, 1999, Exploring MARS: developing indoor and outdooruser interfaces to a mobile augmented reality system, Computer \& Graphics, 23(6): 779-785.

[6] Saito S, Hiyama A, Tanikawa T and Hirose M, 2007, Indoor Marker-based Localization Using Coded Seamless Pattern for Interior Decoration, Proc. IEEE Virtual Reality 2007: 67-74.

[7] Tenmoku R, Kanbara M and Yokoya N, 2003, A wearable augmented reality system using positioning infrastructures and a pedometer, Proc. IEEE Int. Symp. on Wearable Computers: 110-117.

[8] Yoshida H, Ito S and Kawaguchi N, 2006, Evaluation of Pre- Acquisition Methods for Position Estimation System using Wireless LAN, The Third International Conference on Mobile Computing and Ubiquitous Networking (ICMU 2006): 148-155.

[9] Cipolla R, Robertson D and Tordoff B, 2004, Imagebased localization, Proc. Int. Conf. on Virtual System and Multimedia (VSMM2004): 22-29.

[10] Kourogi M, Kurata T and Sakaue K, 2001, A Panorama-based Method of Personal Positioning and Orientation and Its Realtime Applications for Wearable Computers, Proc. Fifth International Symposium on Wearable Computers (ISWC'01): 107-114
[11] Torr $P$ and Zisserman A, 1999, Feature based methods for structure and motion estimation, Int. Work. on Vis. Alg.: 278-295.

[12] Bleser G, Wuest $H$ and Stricker D, 2006, Online camera pose estimation in partially known and dynamic scenes, Proc. Int. Symp. on Mixed and Augmented Reality: 56-65.

[13] Simon G and Berger M, 2002, Pose estimation from planar structures, IEEE Computer Graphics and Applications, vol. 22, No. 6: 46-53.

[14] Hähnel D, Burgard W and Thrun S, 2003, Learning Compact 3D Models of Indoor and Outdoor Environments with a Mobile Robot, Robotics and Autonomous Systems, vol.44, no.1: 15-27.

[15] Jensen B, Weingarten J, Kolski S and Siegwart R, 2005, Laser Range Imaging using Mobile Robots: From Pose Estimation to 3d-Models, Proc. of 1st Range Imaging Research Day: 129-144.

[16] Neubert J, Pretlove J and Drummond T, 2007, Semiautonomous generation of appearance-based edge models from image sequences, Proc. Int. Symp. on Mixed and Augmented Reality: 79-89.

[17] SketchUp, http://www.sketchup.com/.

[18] Ishikawa T, Thangamani K, Kourogi M, Gee A. P, Mayol W, Jung K and Kurata T, 2009, In-Situ 3D Indoor Modeler with a Camera and Self-Contained Sensors, In Proc. HCII2009, LNCS 5622: 454-464.

[19] Langer $M$ and BenHimane S, 2010, An Interactive Vision-based 3D Reconstruction Workflow for Industrial AR Applications, online Proc. Of ISMAR 2010 Workshop on Augmented Reality Super Models.

[20] Arth C, Wagner D, Klopschitz M, Irschara A and Schmalstieg D, 2009, Wide Area Localization on Mobile Phones, In Proc. Int. Symp. on Mixed and Augmented Reality: 73-82.

[21] Klein G and Murray D, 2008, Improving the agility of keyframe-based SLAM, In Proc. 10th European Conference on Computer Vision (ECCV'08).

[22] Irschara A, Zach C, Frahm J.-M and Bischof $\mathrm{H}$, 2009, From structure-from-motion point clouds to fast location recognition, In Conf. on Computer Vision and Pattern Recognition (CVPR).

[23] Kourogi M., Ishikawa T. and Kurata T, 2010, A Method of Pedestrian Dead Reckoning Using Action Recognition, In Proc. IEEE/ION Position, Location and Navigation Symposium (PLANS): 85-89.

[24] Makita $\mathrm{K}$ et al, 2013, Photo-shoot localization of a mobile camera based on registered frame data of virtualized reality models, In S\&T Proc. of ISMAR2013: 273-274.

[25] Bay H, Tuytelaars T and Gool L. V, 2006, SURF: Speeded Up Robust Features, Proc. 9th European Conference on Computer Vision (ECCV'2006), Springer LNCS volume 3951, part 1: 404-417.

[26] Tomasi C and Kanade T, 1991, Detection and tracking of point features, International Journal of Computer Vision.

[27] Vincent T, Nigay L and Kurata T, 2013, Precise pointing techniques for handheld Augmented Reality, in Proc. INTERACT 2013, IFIP-Springer (2013): 122-139.

[28] CAUTIC(Conception Assistée par l'Usage pour les Technologies I'Innovation et le Changement) http://innovacs.upmf-grenoble.fr/innovacs/wpcontent/uploads/2011/12/PPT-CAUTIC-Janv2012.pdf 
[29] Kano N, 1984, Attractive Quality and Must-Be Quality, [in Japanese], The Journal of the Japanese Society for Quality Control, vol.14, No. 2, 147-156.

[30] Sauerwein E, Bailon F, Matzler K and Hinterhuber H, 1996, The Kano model: how to delight your customers, In Preprints Volume I of the IX: International Working Seminar on Production Economics, 313-327. 